\title{
Research Article \\ Remarks on Null Geodesics of Born-Infeld Black Holes
}

\author{
Sharmanthie Fernando \\ Department of Physics and Geology, Northern Kentucky University, Highland Heights, KC 41099, USA \\ Correspondence should be addressed to Sharmanthie Fernando, fernando@nku.edu
}

Received 9 April 2012; Accepted 18 July 2012

Academic Editors: M. Ehrnström, D. Gepner, M. Rasetti, and P. Roy

Copyright (c) 2012 Sharmanthie Fernando. This is an open access article distributed under the Creative Commons Attribution License, which permits unrestricted use, distribution, and reproduction in any medium, provided the original work is properly cited.

We present interesting properties of null geodesics of static charged black holes in Einstein-BornInfeld gravity. These null geodesics represents the path for gravitons. In addition, we also study the path of photons for the Born-Infeld black hole which are null geodesics of an effective geometry. We will present how the bending of light is effected by the non-linear parameter $\beta$ of the theory. Some other properties, such as the horizon radius and the temperature are also discussed in the context of the nonlinear parameter $\beta$.

\section{Introduction}

In Maxwell theory, the field of a point-like charge is singular at the origin. Hence, it has infinite self-energy. To avoid this, Born-Infeld proposed a theory of electrodynamics which is nonlinear in nature which is now known as Born-Infeld electrodynamics [1]. In this theory, the electric filed of a point charge is given as $E_{r}=Q / \sqrt{r^{4}+Q^{2} / \beta^{2}}$ which is regular at the origin. Also, its total energy is finite. Born-Infeld theory has received renewed interest since it turns out to play an important role in string theory. Born-Infeld actions naturally arises in open superstrings and in D-branes [2]. Review articles on the aspects of the Born-Infeld theory in string theory is written by Gibbons [3] and Tseytlin [4].

In this paper, we study null geodesics in the black holes of Einstein-Born-Infeld gravity. The particular black hole in consideration is the nonlinear generalization of the well known Reissner-Nordstrom black hole characterized by charge $Q, M$, and $\beta$. Black hole solutions for Born-Infeld gravity was obtained by Garcia et al. [5] in 1984. Two years later, Demianski [6] also presented a solution known as EBIon. There are many papers written in the literature, addressing various aspects of black holes in Einstein-Born-Infeld gravity. Due to the long list, we will mention only a few recent work here. 
Kruglov published on generalized Born-Infeld electrodynamics in [7]. Thermodynamics of third-order Lovelock-Born-Infeld black holes were studied by peng et al. [8]. Thin shells in Einstein-Born-Infeld theory were studied by Eiroa and Simeone [9]. Linear alanlogs of the Born-Infeld and other nonlinear theories were presented by Milgrom [10]. Test particle trajectories for the static-charged Born-Infeld black hole were discussed by Breton [11]. Gibbons and Herdeiro [12] derived a Melvin Universe-type solution describing a magnetic field. The current author has studied the gravitational, scalar, and Dirac perturbations of the Born-Infeld black holes in [13-15], respectively. Non-abelian black hole solutions to BornInfeld gravity were presented by Mazharimousavi et al. [16]. Hairy mass bound in the Einstein-Born Infeld black holes were given by Myung and Moon [17].

\section{Static Charged Black Hole in Einstein-Born-Infeld Gravity}

The Einstein-Born-Infeld gravity is given by the action

$$
S=\int d^{4} x \sqrt{-g}\left[\frac{R}{16 \pi G}+L(F)\right]
$$

where $L(F)$ is a function of the field strength $F_{\mu \nu}$ given as

$$
L(F)=4 \beta^{2}\left(1-\sqrt{1+\frac{F^{\mu \nu} F_{\mu \nu}}{2 \beta^{2}}}\right)
$$

Here, $\beta$ has dimensions length ${ }^{-2}$ and $G$ length ${ }^{2}$. We will assume $16 \pi G=1$ in the rest of the paper. Note that when the nonlinear parameter $\beta \rightarrow \infty$, the function $L(F)$ approaches the one for Maxwell's electrodynamics given by $-F^{2}$.

The static-charged black hole solution with spherical symmetry for the above action in (2.1) is given as

$$
d s^{2}=-f(r) d t^{2}+f(r)^{-1} d r^{2}+r^{2}\left(d \theta^{2}+\sin ^{2}(\theta) d \varphi^{2}\right)
$$

with

$$
f(r)=1-\frac{2 M}{r}+\frac{2 \beta^{2} r^{2}}{3}\left(1-\sqrt{1+\frac{Q^{2}}{r^{4} \beta^{2}}}\right)+\frac{4 Q^{2}}{3 r^{2}}{ }_{2} F_{1}\left(\frac{1}{4}, \frac{1}{2}, \frac{5}{4},-\frac{Q^{2}}{\beta^{2} r^{4}}\right) .
$$

Here ${ }_{2} F_{1}$ is the hypergeometric function.

The electric field is given by

$$
F_{t r}=E(r)=-\frac{Q}{\sqrt{r^{4}+Q^{2} / \beta^{2}}}
$$


In this case, the $L(F)$ reduces to

$$
L(F)=4 \beta^{2}\left(1-\sqrt{1-\frac{E^{2}}{\beta^{2}}}\right) .
$$

One can observe that there is an upper bound for the electric field as $|E| \leq \beta$. This is one of the leading characteristics of Born-Infeld electrodynamics which leads to finite self-energy of the electron as compared to Maxwell electrodynamics.

When the non-linear parameter $\beta \rightarrow \infty$, the function $f(r)$ approaches

$$
f(r)_{\mathrm{RN}}=1-\frac{2 M}{r}+\frac{Q^{2}}{r^{2}}
$$

which is the metric function $f(r)$ for the static charged black hole in Einstein-Maxwell gravity which is known as the Reissner-Nordstrom black hole. Reissner-Nordstrom black hole has horizons at

$$
r_{ \pm}=M \pm \sqrt{M^{2}-Q^{2}}
$$

For the Born-Infeld black hole, near the origin, the function $f(r)$ has the behavior as,

$$
f(r) \approx 1-\frac{(2 M-A)}{r}-2 \beta Q+\frac{2 \beta^{2}}{3} r^{2}+\frac{\beta^{3}}{5} r^{4}
$$

Here,

$$
A=\frac{1}{3} \sqrt{\frac{\beta}{\pi}} Q^{3 / 2} \Gamma\left(\frac{1}{4}\right)^{2}
$$

Hence, for $2 M>A, f(r) \rightarrow-\infty$ for small $r$ and for $2 M<A, f(r) \rightarrow+\infty$ for small $r$. When $r \rightarrow \infty$, for all values of $M$ and $A, f(r) \rightarrow 1$. In fact graphically, we will show that for various values of $M, Q$ and $\beta$, that $f(r)$ could have two roots, one root, or none. Extreme black holes are possible when $f(r)=0$ and $f^{\prime}(r)=0$, leading to the horizon radius as

$$
r_{\mathrm{ex}}=\frac{\sqrt{4 \beta^{2} Q^{2}-1}}{2 \beta} .
$$

It is clear that extreme black holes exist only if $Q \beta>1 / 2$. In Figure 1, the function $f(r)$ is plotted for both $Q \beta<1 / 2$ and $Q \beta>1 / 2$.

In Figure 2, the horizon radius $r_{+}$is computed for various values of $\beta$. The horizon radius decreases with $\beta$. For the same values of $M$ and $Q$, the horizon radius for the ReissnerNordstrom black hole is given by $r_{+}=1.42765$. 


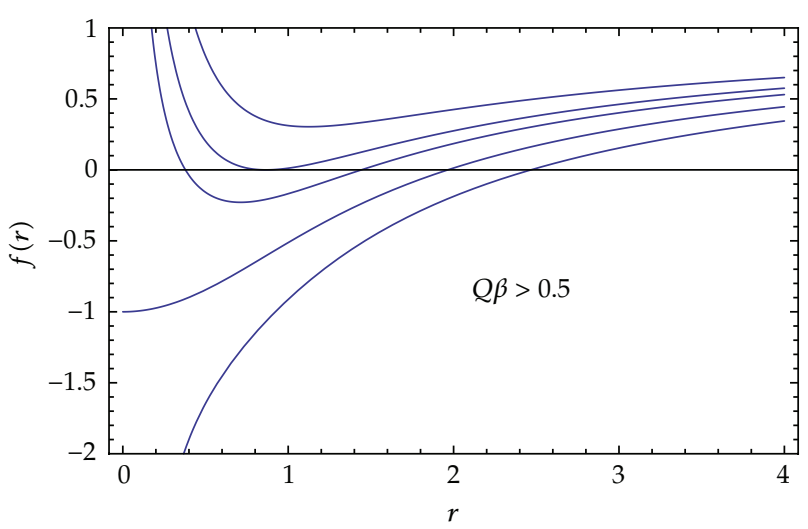

(a)

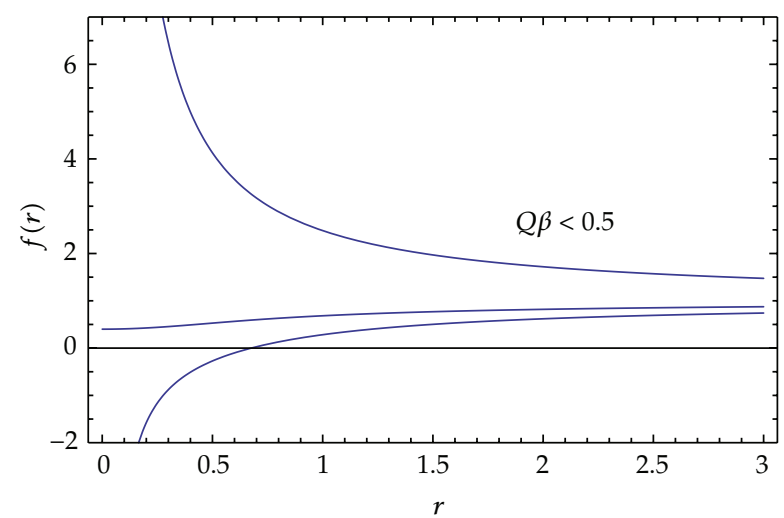

(b)

Figure 1: The function $f(r)$ for various values of $M$ for fixed values of $Q$ and $\beta$. In the top graph (a), $Q=1$ and $\beta=1$. In the bottom graph (b), $Q=0.3$ and $\beta=1$.

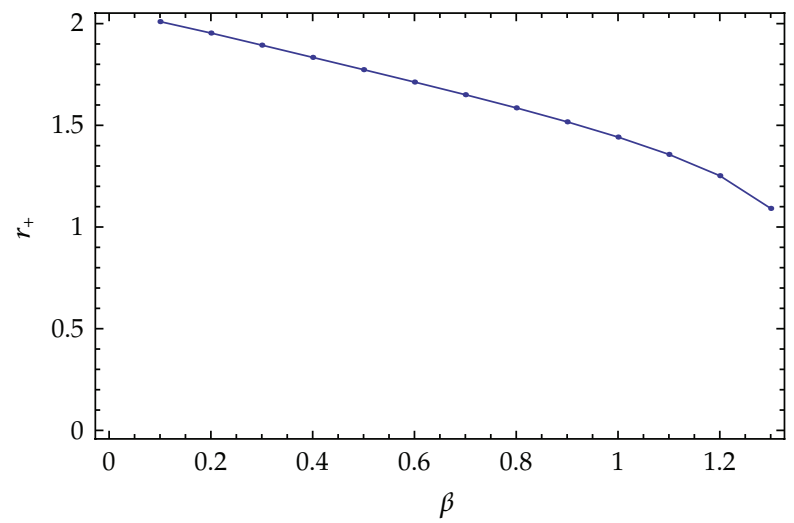

Figure 2: The horizon radius $r_{+}$for various values of $\beta$. Here, $Q=1$ and $M=1.06405$. 


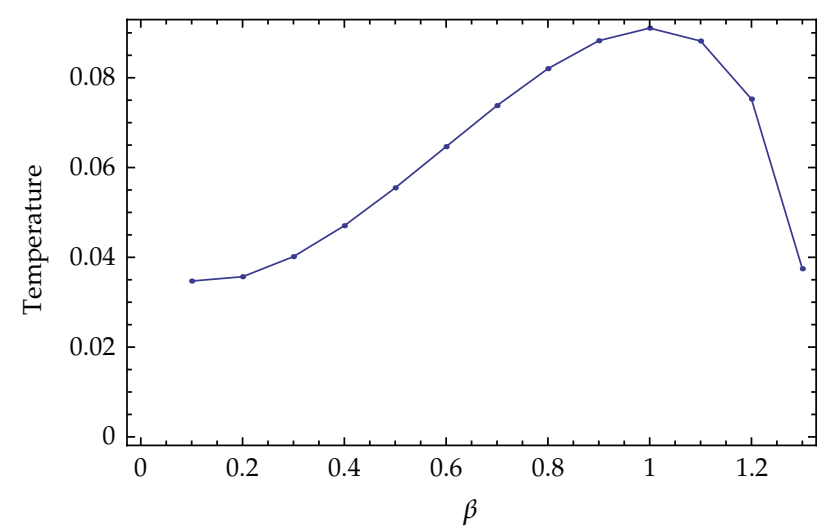

Figure 3: The temperature $T$ for various values of $\beta$. Here, $Q=1$ and $M=1.06405$.

The Hawking temperature of the Born-Infeld black hole is given by

$$
T=\frac{1}{4 \pi}\left[\frac{1}{r_{+}}+2 \beta\left(r_{+} \beta-\frac{\sqrt{\left(Q^{2}+r_{+}^{2} \beta^{2}\right)}}{r_{+}}\right)\right] .
$$

Here, $r_{+}$is the event horizon of the black hole such that $f(r)=0$. In Figure 3, a graph for temperature versus $\beta$ is plotted. It seems the temperature has a maximum before decreasing for this particular values of $M$ and $Q$.

To compare the temperature of te Born-Infled black hole to the Reissner-Nordstrom black hole, one can compute the temperature for the outer horizon given in (2.8) as,

$$
T_{\mathrm{RN}}=\frac{1}{4 \pi}\left(-\frac{2 Q^{2}}{r_{+}}+\frac{2 M}{r_{+}}\right) .
$$

For the same values of $M$ and $Q$ given in the Figure 3, the temperature for the ReissnerNordstrom black hole is given as $T_{\mathrm{RN}}=0.356786$. Hence, the Reissner-Nordstrom black hole is "hotter" compared to its counterpart in Born-Infeld gravity. The zeroth and the first law of the Born-Infeld black holes are discussed by Rasheed in [18].

\section{Null Geodesics of the Born-Infeld Black Hole}

The motion of graviton in the background of the Born-Infeld black hole is given by the null geodesics. In general, it is also the path of photons, which is not the case here. This will be discussed in Section 4.

The geodesic equations for the Born-Infeld black hole can be derived from the Lagrangian equation

$$
\mathcal{L}=-\frac{1}{2}\left(-f(r)\left(\frac{d t}{d \tau}\right)^{2}+\frac{1}{f(r)}\left(\frac{d r}{d \tau}\right)^{2}+r^{2}\left(\frac{d \theta}{d \tau}\right)^{2}+r^{2} \sin ^{2} \theta\left(\frac{d \phi}{d \tau}\right)^{2}\right) .
$$


Here, $\tau$ is an affine parameter along the geodesics. The derivation is clearly given in the well known book by Chandrasekhar [19]. Therefore, we will skip some of the details here. Since the Born-Infeld black holes have two Killing vectors $\partial_{t}$ and $\partial_{\phi}$, there are two constants of motion which can be labeled as $E$ and $L$ given as

$$
\begin{gathered}
f \dot{t}=E, \\
r^{2} \sin ^{2} \theta \dot{\phi}=L .
\end{gathered}
$$

Here, we will choose $\theta=\pi / 2$ and $\dot{\theta}=0$ as the initial conditions, which leads to, $\ddot{\theta}=0$. Hence, $\theta$ will remain at $\pi / 2$ and the geodesics will be described in an invariant plane at $\theta=\pi / 2$. From (3.2),

$$
\begin{gathered}
r^{2} \dot{\phi}=L \\
f(r) \dot{t}=E .
\end{gathered}
$$

By substituting these values to the Lagrangian in (3.1), one obtains the geodesics as,

$$
\dot{r}^{2}+f(r)\left(\frac{L^{2}}{r^{2}}+h\right)=E^{2}
$$

Here, $2 \mathcal{L}=h . h=0$ corresponds to null geodesics and $h=1$ corresponds to time-like geodesics. Note that (3.5) can be written as, $\dot{r}^{2}+V_{\text {eff }}=E^{2}$, with the effective potential,

$$
V_{\mathrm{eff}}=\left(\frac{L^{2}}{r^{2}}+h\right) f(r)
$$

From (3.3) and (3.5), one can get a relation between $\phi$ and $r$ as follows:

$$
\frac{d \phi}{d r}=\frac{L}{r^{2}} \frac{1}{\sqrt{\left(E^{2}-V_{\text {eff }}\right)}}
$$

\subsection{Effective Potential for Null Geodesics}

With $h=0$,

$$
\mathrm{V}_{\text {eff }}=f(r) \frac{L^{2}}{R(r)^{2}}
$$

We will only consider the gravitons with nonzero angular momentum here. In Figure 4, the $V_{\text {eff }}$ is given for various values of $\beta$. The height for the Born-Infled black hole is shorter in comparison with the Reisnner-Nordstrom black hole.

In Figure 5, the effective potential is plotted for three different energy levels, $E_{1}, E_{c}$ and $E_{2}$. This gives different scenarios of motion of the particles which are described below. 


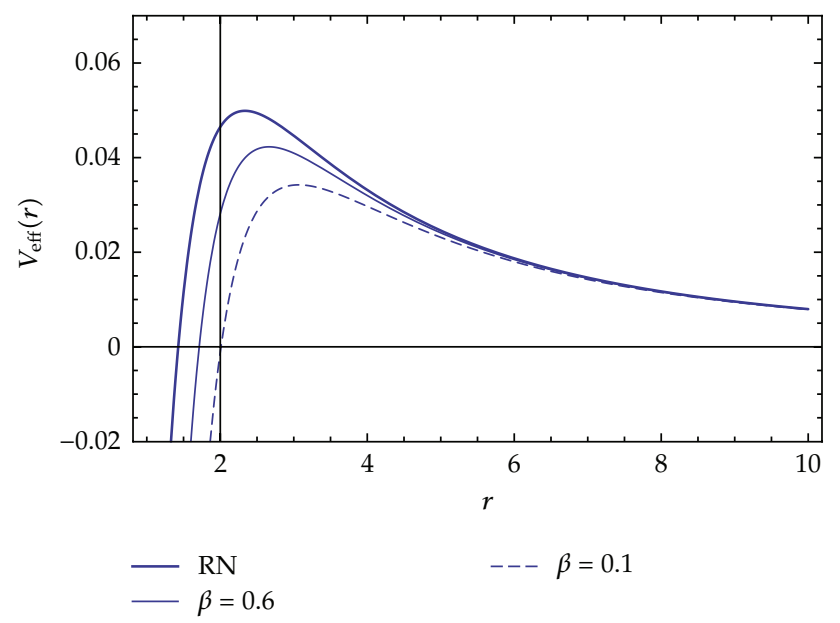

Figure 4: The graph shows the relation of $V_{\text {eff }}$ with the parameter $\beta$. Here, $M=1.06405, L=1$ and $Q=1$.

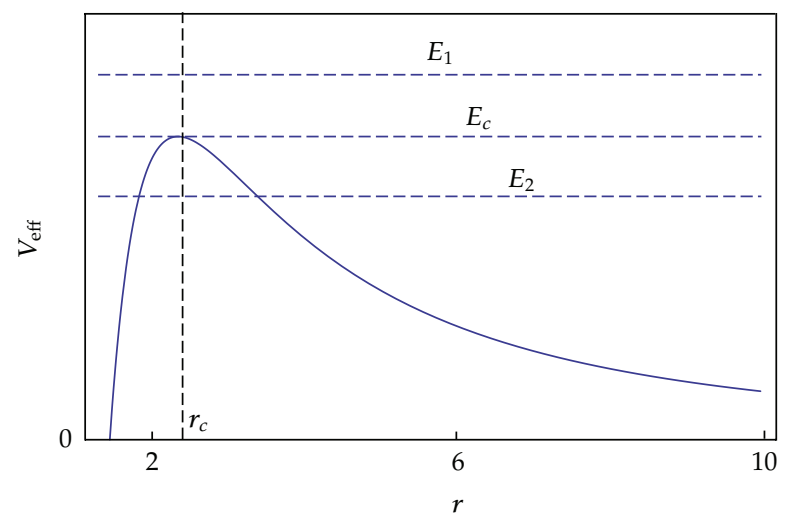

Figure 5: The graph shows the relation of $V_{\text {eff }}$ with the energy $E$. Here, $M=1.06405, \beta=1, Q=1$ and $L=1$.

Case $1\left(E=E_{c}\right)$. Here, $E^{2}-V_{\text {eff }}=0$ leads to circular orbits. From the nature of the potential at $r=r_{c}$, one can conclude that these are unstable circular orbits.

Case $2\left(E=E_{2}\right)$. Here, the motion is possible only in the regions where, $E^{2}-V_{\text {eff }} \geq 0$.

Case $3\left(E=E_{1}\right)$. Since $E^{2}-V_{\text {eff }} \geq 0$ and $\dot{r}>0$ for all $r$ values, motion is possible for all $r$ values.

For Case 1, one can compute the radius of the circular orbits. The conditions for the circular orbits are

$$
\begin{gathered}
\dot{r}=0 \Longrightarrow V_{\text {eff }}=E_{c}^{2}, \\
\frac{d V_{\text {eff }}}{d r}=0 .
\end{gathered}
$$

From (3.10), $r_{c}$ can be computed numerically. It is given in Figure $6 . r_{c}$ decrease as $\beta$ increases. 


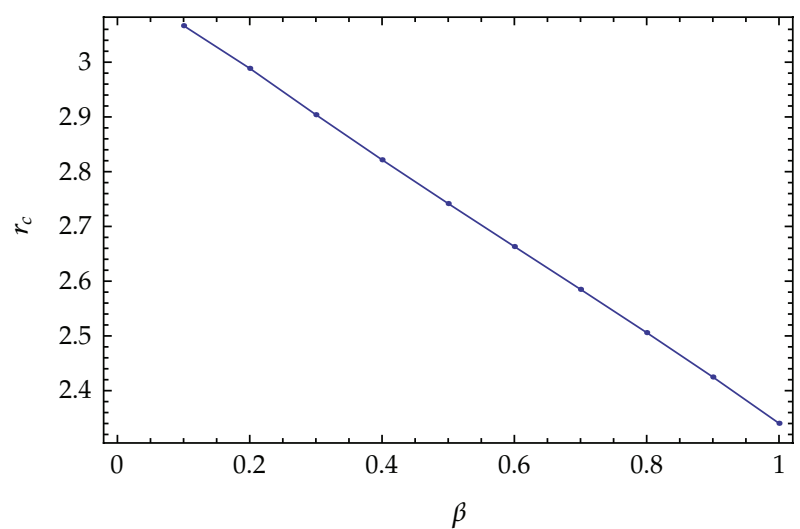

Figure 6: The graph shows the critical radius $r_{c}$ for the Born-Infeld black hole as a function of the nonlinear parameter $\beta$. Here, $M=1.06405$ and $Q=1$.

\section{Null Geodesics of the Effective Geometry}

In general, the motion of photons are represented by the null geodesics of the spacetime. However, in nonlinear electrodynamics, the path of the photons are not given by the null geodesics of the background metric. The path is given by null geodesics of an effective geometry generated by the self-interaction of the electromagnetic field. This effective geometry depends on the particular nonlinear theory considered, and in Einstein-Born-Infeld gravity, the effective geometry is given by

$$
d s^{2}=-h(r) d t^{2}+g(r)^{-1} d r^{2}+R(r)^{2}\left(d \theta^{2}+\sin ^{2}(\theta) d \phi^{2}\right),
$$

where

$$
\begin{gathered}
\omega(r)=1+\frac{Q^{2} b^{2}}{r^{4}}, \\
g(r)=f(r) \omega(r)^{-1 / 2}, \\
h(r)=f(r) \omega(r)^{1 / 2}, \\
R(r)^{2}=r^{2} \omega(r)^{-1 / 2} .
\end{gathered}
$$

Hence, gravitational lensing, which is related to the bending of light around the black hole can be computed from the knowledge gained from null geodesics of the effective geometry. The derivation leading to the null geodesics are similar to the one given in Section 3. Since the symmetries are the same, there will be two conserved quantities as,

$$
\begin{gathered}
R(r)^{2} \dot{\phi}=L \\
f(r) \dot{t}=E .
\end{gathered}
$$




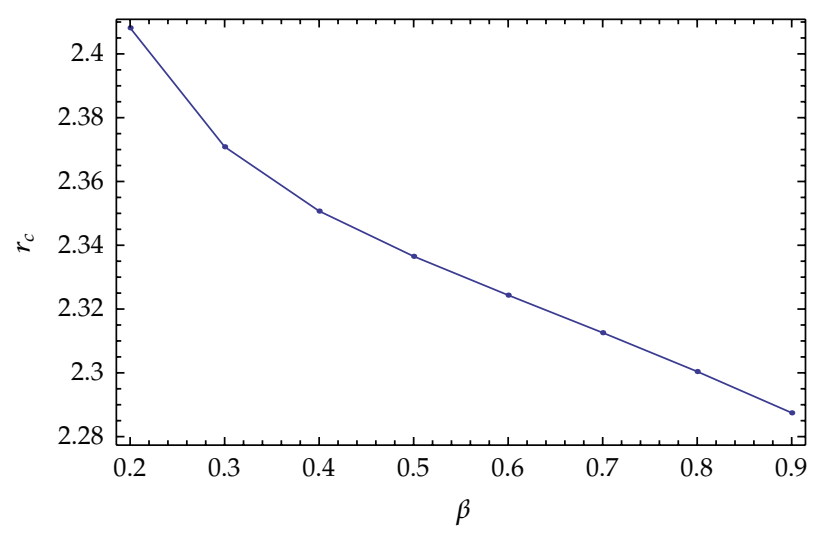

Figure 7: The graph shows the critical radius $r_{c}$ for the Born-Infeld black hole as a function of the nonlinear parameter $\beta$. Here, $M=1.06405$ and $Q=1$.

The equations can be given as $\dot{r}^{2}+V_{\text {eff }}=0$, where the effective potential, which depends on $E$, and $L$ is given as follows:

$$
V_{\mathrm{eff}}=L^{2} \frac{g(r)}{R(r)^{2}}-E^{2} \frac{g(r)}{h(r)}
$$

\subsection{Circular Orbits}

The conditions for the circular orbits are

$$
\begin{gathered}
\dot{r}=0 \Longrightarrow V_{\text {eff }}=0, \\
\frac{d V_{\mathrm{eff}}}{d r}=0 .
\end{gathered}
$$

These two conditions lead to the equation

$$
h(r) R(r)^{2^{\prime}}-h(r)^{\prime} R(r)^{2}=0 .
$$

One can obtain a solution for the circular orbits at $r=r_{c}$ by solving (4.7) numerically.

From Figure 7 , it is clear that $r_{c}$ decreases for increasing $\beta$ values. The circular orbit at $r=r_{c}$ are unstable. The radius of the circular orbit is related to $E$ and $L$ as

$$
\frac{E_{c}^{2}}{L_{c}^{2}}=\frac{h\left(r_{c}\right)}{R\left(r_{c}\right)^{2}} .
$$

When $\beta \rightarrow \infty$ and $Q \rightarrow 0, r_{c} \rightarrow 3 M$ which is the radius of the unstable circular orbit of the Schwarzschild black hole [19]. 


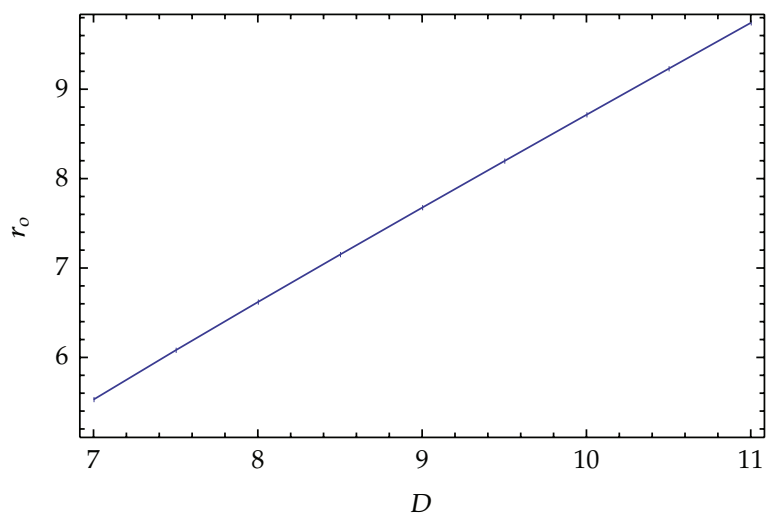

Figure 8: The graph shows the closest approach $r_{o}$ for the effective geometry of the Born-Infeld black hole as a function of the impact parameter $D$. Here, $M=1.06405, Q=1$ and $L=1$.

\subsection{Bending of Light}

To compute the angle of bending of light, first, let us compute the closest approach $r_{o}$. It is defined by the solutions to the equation $d r / d \phi=0$. From (4.3) and (4.5)

$$
\left(\frac{1}{R(r)^{2}} \frac{d r}{d \phi}\right)^{2}=\frac{g(r)}{R(r)^{2}}-\frac{E^{2}}{L^{2}} \frac{g(r)}{h(r)}
$$

Since $E^{2} / L^{2}=1 / D^{2}$, where $D$ is the impact parameter, the above equation $d r / d \phi=0$ simplifies to,

$$
D^{2} h(r)-R(r)^{2}=0
$$

For various values of $D$ and $\beta,(4.10)$ can be solved numerically to obtain $r_{o}$. In Figure 8 and Figure 9, the graph $r_{o}$ versus $D$ and $\beta$ is given. For large $D, r_{o}$ becomes larger as expected. For large $\beta, r_{O}$ decreases.

Gravitational lensing of the photons in the Born-Infeld black hole was studied by Eiroa [20]. In an interesting paper by Amore [21], analytical expression for the bending angle was derived. Here, we will use that expression which is given as

$$
\alpha=\frac{4 M}{r_{o}}+\left(\frac{24 M^{2}}{\pi r_{o}^{2}}-\frac{3 \pi Q^{2}}{4 r_{o}^{2}}\right)+\left(\frac{160 M^{3}}{\pi^{2} r_{o}^{2}}-\frac{9 M Q^{2}}{r_{o}^{3}}\right)+O\left[\frac{1}{r_{o}^{4}}\right]
$$

One can compute the bending angle as a function of $D$ and $\beta$ which is presented in Figures 10 and 11. The bending is grater for large $D$ as expected. The angle $\alpha$ increases as $\beta$ increases. 


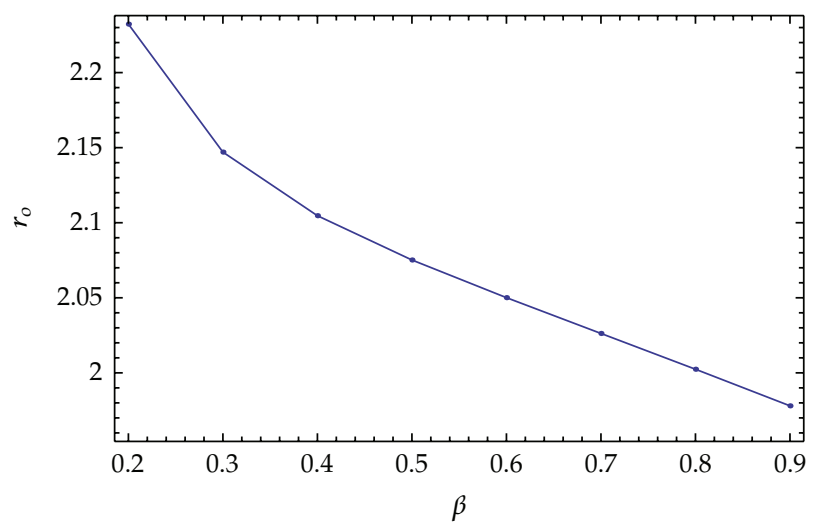

Figure 9: The graph shows the closest approach $r_{o}$ for the effective geometry of the Born-Infeld black hole as a function of the nonlinear parameter $\beta$. Here, $D$ is kept fixed at 4.56. Also, $M=1.06405, Q=1$.

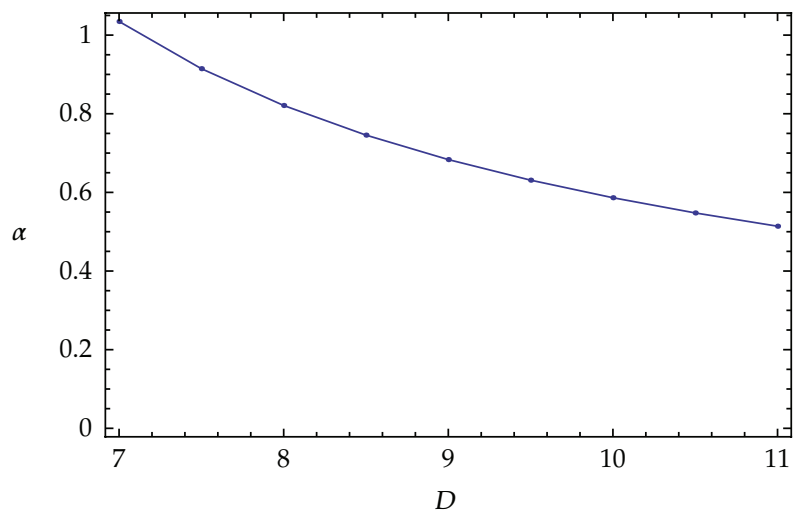

Figure 10: The graph shows the bending angle $\alpha$ for the Born-Infeld black hole as a function of the impact parameter $D$. Here, $M=1.06405, Q=1$ and $L=1$.

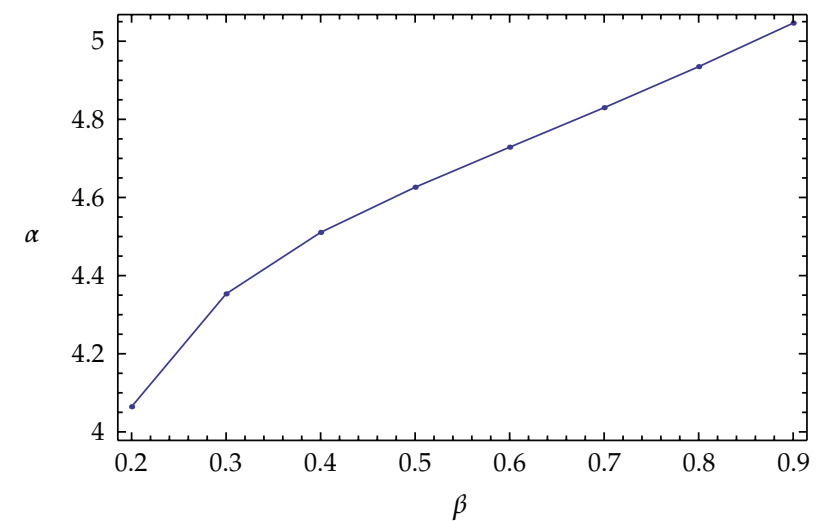

Figure 11: The graph shows the bending angle $\alpha$ for the Born-Infeld black hole as a function of the $\beta$. Here, the impact parameter is kept fixed at $D=4.56$. Also, $M=1.06405$ and $Q=1$. 


\section{Conclusions}

In this paper, we have done a detailed study of null geodesics of the Born-Infeld black hole for the gravitons and the photons. Unlike in other cases, null geodesics of the black hole is not the path of the photons. Path for photons are given by an effective geometry. We have studied the bending of light and showed that the bending angle increases with the nonlinear parameter $\beta$. On the other hand, the bending angle decreases with the impact parameter $D$.

We have also discussed the thermodynamics of the black hole and showed how the temperature vary with $\beta$. For the same mass and the charge, the corresponding ReissnerNordstrom black hole is "hotter".

The horizon radius also seems to decrease with the increasing $\beta$ for the particular mass and charge considered.

In extending this work, it would be interesting to study massive test particles around the Born-Infeld black hole.

\section{References}

[1] M. Born and L. Infeld, "Foundations of the new field theory," Proceedings of the Royal Society A, vol. A144, pp. 425-451, 1934.

[2] R. G. Leigh, "Dirac-Born-Infeld action from Dirichlet sigma model,” Modern Physics Letters A, vol. 4, Article ID 2767, 1989.

[3] G. W. Gibbons, "Aspects of Born-Infeld theory and string/M-theory," http://arxiv.org/abs/hep-th/ 0106059.

[4] A. A. Tseytlin, "Born-Infeld action, supersymmetry and string theory," http://lanl.arxiv.gov/abs / hep-th/9908105.

[5] A. Garcia, H. Salazar, and J. F. Plebanski, "Type-D solutions of the Einstein and Born-Infeld nonlinearelectrodynamics equations," Nuovo Cimento, vol. 84, pp. 65-90, 1984.

[6] M. Demianski, "Static electromagnetic geon," Foundations of Physics, vol. 16, no. 2, pp. 187-190, 1986.

[7] S. I. Kruglov, "On generalized Born-Infeld electrodynamics," Journal of Physics A, vol. 43, Article ID 375402, 2010.

[8] L. Peng, Y. Rui-Hong, and Z. De-Cheng, "Thermodynamics of third order lovelock-Born-Infeld Black holes," Communications in Theoretical Physics, vol. 56, pp. 845-850, 2011.

[9] E. F. Eiroa and C. Simeone, "Thin shells in Einstein-Born-Infeld theory," AIP Conference Proceedings, vol. 1458, pp. 383-386, 2012.

[10] M. Milgrom, "Practically linear analogs of the Born-Infeld and other nonlinear theories," Physical Review D, vol. 85, Article ID 105018, 2012.

[11] N. Breton, "Born-Infeld generalization of the Reissner-Nordstrom black hole," http:/ /lanl.arxiv.gov/ abs/gr-qc/010922.

[12] G. W. Gibbons and C. A. R. Herdeiro, "The Melvin universe in Born-Infeld theory and other theories of nonlinear electrodynamics," Classical and Quantum Gravity, vol. 18, pp. 1677-1690, 2001.

[13] S. Fernando, "Gravitational perturbation and quasi-normal modes of charged black holes in EinsteinBorn-Infeld gravity," General Relativity and Gravitation, vol. 37, pp. 585-604, 2005.

[14] S. Fernando and C. Holbrook, "Stability and quasi normal modes of charged born-infeld black holes," International Journal of Theoretical Physics, vol. 45, pp. 1630-1645, 2006.

[15] S. Fernando, "Decay of massless dirac field around the Born-Infeld black hole," International Journal of Modern Physics A, vol. 25, pp. 669-684, 2010.

[16] S. H. Mazharimousavi, M. Halisoy, and Z. Amirabi, "New non-Abelian black hole solutions in BornInfeld gravity," Physical Review D, vol. 78, Article ID 064050, 2008.

[17] Y. S. Myung and T. Moon, "Hairy mass bound in the Einstein-Born-Infeld black hole," http://lanl .arxiv.org/abs/1201.1173.

[18] D. A. Rasheed, "Non-linear electrodynamics: zeroth and First Laws of Black Hole Mechanics," http:/ / lanl.arxiv.org/abs/hep-th/9702087. 
[19] S. Chandrasekhar, The Mathematical Theory of Black Holes, Oxford University Press, 1992.

[20] E. F. Eiroa, "Gravitational lensing by Einstein-Born-Infeld black holes," Physical Review D, vol. 73, no. 4, Article ID 043002, 2006.

[21] P. Amore, S. Arceo, and F. M. Fernandez, "Analytical formulas for gravitational lensing: higher order calculation," Physical Review D, vol. 74, Article ID 083004, 2006. 


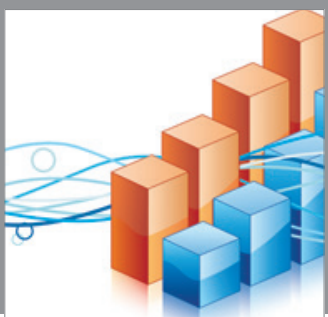

Advances in

Operations Research

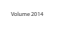

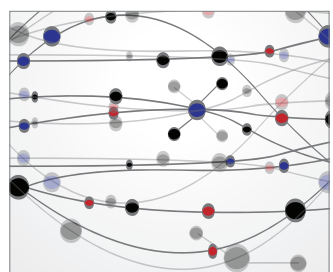

\section{The Scientific} World Journal
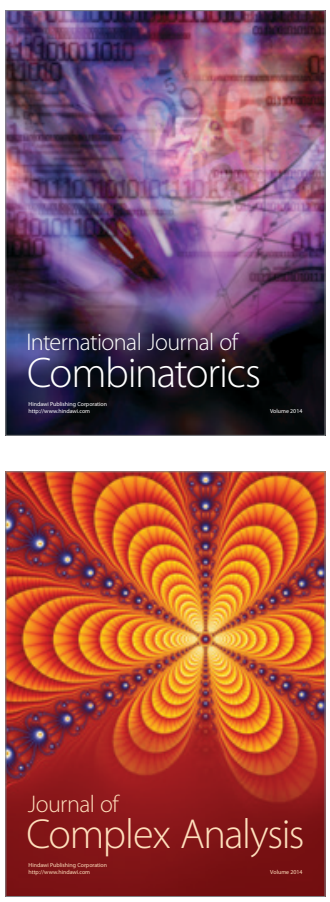

International Journal of

Mathematics and

Mathematical

Sciences
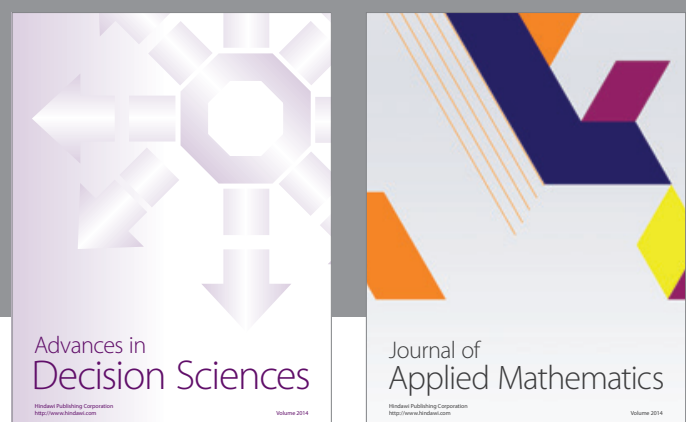

Journal of

Applied Mathematics
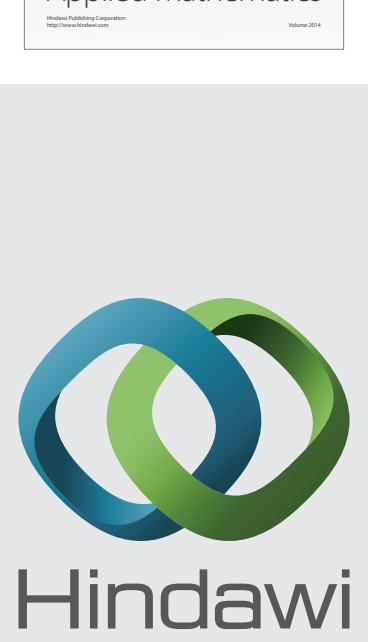

Submit your manuscripts at http://www.hindawi.com
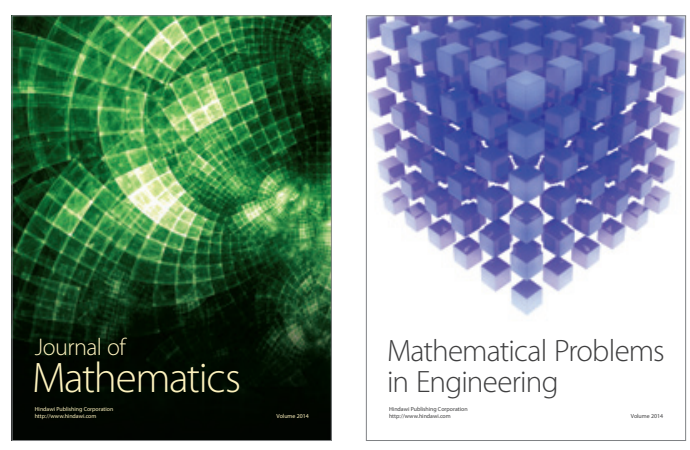

Mathematical Problems in Engineering
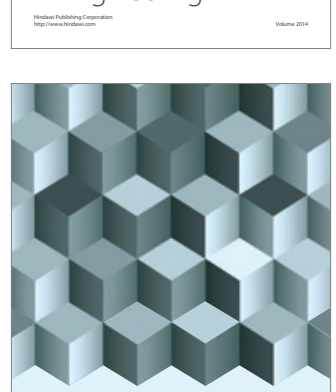

Journal of

Function Spaces
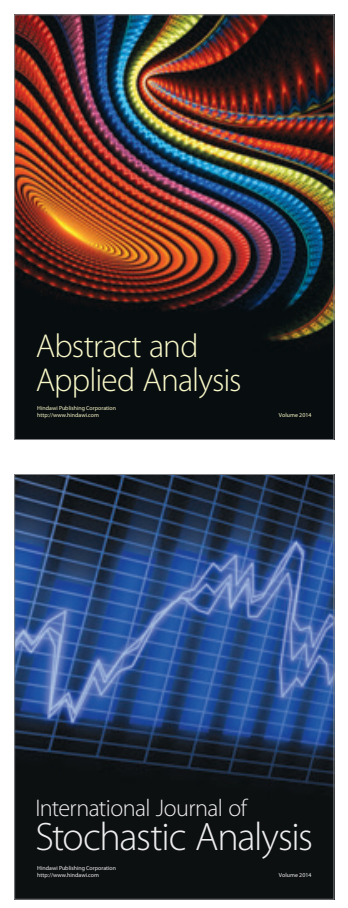

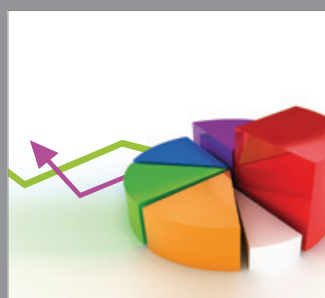

ournal of

Probability and Statistics

Promensencen
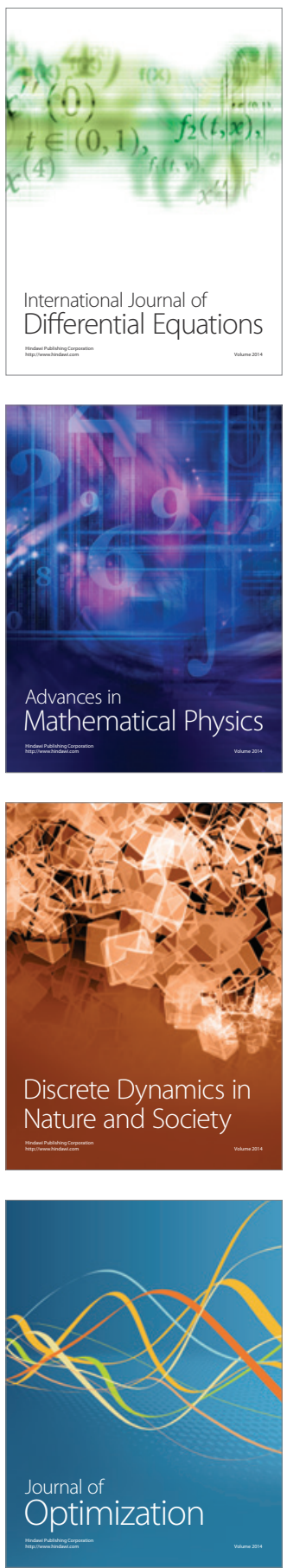